\title{
ULTRASOUND ATTENUATION COEFFICIENT IN THE FETAL LIVER AS A FUNCTION OF GESTATIONAL AGE
}

\author{
Paul L. Carson, Charles R. Meyer, Edward H. Chiang, \\ ROGER G. FAIX and TERRI I. MARKS \\ University of Michigan Medical Center, Ann Arbor, MI 48109-0553
}

(Received 24 July 1989; in final form 20 November 1989)

\begin{abstract}
An apparent increase in the ultrasound attenuation coefficient per unit frequency, $\alpha_{f}$, of fetal liver as a function of gestational age has been observed. Measurements were made in utero with a 25 megasample/sec RF digitizer and a real time ultrasound system with a $5 \mathrm{MHz}$ scan head. A precise measurement of $\alpha_{f}$ was employed in which the intercept was tied to 0 at a frequency of 0. In 178 examinations of normal pregnancies, the linear regression of the $\alpha_{f}$ increased $26 \%$ between 26 and 40 weeks gestation. This statistically significant increase ( $p$ $<0.0001)$ is consistent with several observations, those of Parker et al. of increased attenuation in liver when glycogen is added, the increasing glycogen storage in the liver before birth, and our own pre- and postnatal measurements reported elsewhere. A noninvasive assay for glycogen content would have important applications in medicine and biomedical science. However, an increase in measurement accuracy and precise correlation with glycogen content will be required to make meaningful predictions in individual cases, as opposed to the present statistical trends.
\end{abstract}

Key Words: Abdomen, Acoustics, Diagnostic imaging, Fetal development, Fetal growth retardation, Fetal monitoring, Gluconeogenesis, Glycogen, Human, Liver, Liver function tests, Liver Glycogen, Metabolic disease, Nutrition, Nutritional disorders, Prenatal diagnosis, Ultrasonic diagnosis, Ultrasonic scanning, Ultrasonic tissue characterization, Ultrasonics.

\section{BACKGROUND}

In the summer of 1986, an apparent increased attenuation by the fetal liver as a function of gestational age was observed in early data from a study of fetal lung maturity in which liver was used as an attenuation and echogenicity comparison for the lung. Shortly after, Duerinckx et al. $(1986,1988)$ indicated how strongly ultrasound attenuation correlated with liver function tests in their study of adults with and without liver disease. This suggested thinking about the greatest changes, normally occurring in the liver during third trimester pregnancies and that led to consideration of the great storage of glycogen near term. If, indeed, glycogen changes were responsible for changes in ultrasound attenuation and a noninvasive assay could be developed for glycogen content in the fetus, and even children and adults, that would be quite exciting. There is no such noninvasive assay at present and most third trimester concerns involve

Address reprint requests to: Paul L. Carson, Ph.D., University of Michigan Hospitals, Department of Radiology, Kresge III, Ann Arbor, MI 48109-0553. intrauterine growth retardation or other nutritional disorders. Since glycogen stores can be depleted in 12 hours of fasting or a few hours of labor and birth (Shelly 1961), glycogen concentration should be an immediate and sensitive indicator of nutritional sufficiency. Our fetal measurements and others' measurements to verify the hypothesis of glycogen-caused variations in liver attenuation (Parker et al. 1988; Tuthill et al. 1989) appear promising as discussed below.

\section{METHODS}

Measurements were made in utero with an ATL Mark 100 mechanical real time system, with a $5 \mathrm{MHz}$ scan head, 25 megasample/sec RF digitization, and 2-4 Mbytes of liver data per subject. The $10 \mathrm{~mm}$ diameter transducers in the model 420 scan head were focused at $3.2 \mathrm{~cm}$ and had a $6 \mathrm{~dB}$ bandwidth of $2.2 \mathrm{MHz}$.

The slope of the ultrasonic attenuation coefficient with frequency, or the attenuation coefficient per unit frequency, $\alpha_{f}$, was measured by the method 
of Kuc and Schwartz (1979), as implemented by Meyer et al. (1984). Attenuation,

$$
A(f)=\exp -(a(f) \Delta z),
$$

was measured from the RF signal at each frequency over a $1.7 \mathrm{MHz}$ bandwidth and each location between $7.5 \mathrm{~mm}$ segments of tissue. Initial corrections for beam diffraction and swept gain were made by scanning a homogeneous attenuation and backscatter test object immediately after scanning the subject and with the same swept gain and other settings. The difference between the natural $\log$ of the measured power spectra from the tissue and test object is computed from the $n$th segment. Pairs of these quantities, separated by $D$ segments, are subtracted, then all these difference log spectra are averaged and fit with least squares linear fit, where the resulting slope and intercept are $\alpha_{f}$ and $\alpha_{0}$. That is, the attenuation coefficient spectrum is:

$$
a(f)=\alpha_{f} f+\alpha_{0}=\left(\ln A\left(z_{2}\right)-\ln A\left(z_{1}\right)\right) / D \Delta z .
$$

In a few cases an attenuator setting was changed between scanning the subject and the reference test object. Theoretical diffraction corrections have been proposed and applied by numerous authors, e.g., Cloostermans and Thijssen (1983), but these corrections have not generally worked well. This direct, experimental method should be no worse than the simple, linear theories and might correct in part for some system imperfections and physical phenomena that are not accounted for in the available theories. The test objects scanned as a reference for diffraction corrections were homogeneous attenuation test objects obtained from Radiation Measurements, Inc. Great attention was paid to obtaining the most precise and accurate estimate from the small tissue volumes accessed in the fetal lung and liver.

In the actual human subject data, rather than fitting the slope and intercept of a line to the natural $\log$ of attenuation per $\mathrm{cm}$ at each frequency, the intercept was fixed to zero attenuation coefficient at zero frequency. That is, a fixed value of zero was taken for $\alpha_{0}$. This gave greatly reduced variance of the attenuation coefficient slope estimate, $\alpha_{f}$, on test objects (Carson and Meyer 1986), as illustrated in Fig. 1 and Table 1. The large, factor of 6 , reduction in the SEM of $\alpha_{f}$ is consistent with the predicted ratio of the zero to nonzero intercept variances:

$$
\sigma_{z}^{2} / \sigma_{n z}^{2}=\sum_{i=1}^{N}\left(f_{i}-\bar{f}\right)^{2} / \sum_{i=1}^{N}\left(f_{i}\right)^{2},
$$

where $f_{i}$ and $\bar{f}$ are the $i$ th and mean measurement frequencies, respectively.
Unfortunately, this zero-intercept model can also reduce the reported contrast, or differences in attenuation coefficient slope of two tissue classes, when the linear, zero-intercept model is incorrect and the true slope over the limited range of measured frequencies is varying more than the attenuation coefficient divided by the frequency (Carson et al. 1988). The literature is mixed on the issue of how good the zero-intercept hypothesis is for liver attenuation coefficient. In certain pathologies and with adequate bandwidth and sample volume, at least a two parameter linear fit is of value and a power law dependence on frequency may also be worthwhile (Lin et al. 1987; Lizzi et al. 1988; Parker et al. 1984). In normal liver, however, a good fit is usually obtained with a linear model over the range of typical diagnostic frequencies (Goss et al. 1979; Nicholas 1982). In fact, the zero intercept model is a good representation of normal liver for $1-8 \mathrm{MHz}$ data (Lin et al. 1987). We currently believe the statistical stability of the single-parameter, zero-intercept model warrants its use at least in normal fetal liver. Some of the confusion regarding the use of $\alpha_{f}$ in the zero-intercept method might be alleviated by referring in this case to $\alpha_{f}$, not as the attenuation coefficient slope, but as the attenuation coefficient divided by the measurement frequency, i.e., the attenuation coefficient per unit frequency.

Calibration and comparison measurements on several test objects are presented in Table 1. The through transmission values in the last column of Table 1 were measured reproducibly in our laboratory by substitution of the test object material for water in the path between a transducer and a flat steel plate. However, that geometry, which allows inclusion of some of the forward-scattered energy, does not always provide the same result as would be obtained by an accurate attenuation measurement based purely on the change in backscattered energy with range. The means between the transmission and the nominal values (column 2) were therefore chosen as the accepted values. With this selection of accepted values $\alpha_{f}$, the mean backscatter measurement $\alpha_{f m}$ $=0.27$ of test object $\mathrm{B}$ agrees closely with the accepted $\alpha_{f}=0.26$. So does the depth dependent backscatter measurement of $\alpha_{f z} z+\alpha_{f 0}=0.27$ if we assume that the range-dependent measurements are correct at the $4.5 \mathrm{~cm}$ mean range of the Regions of Interest (ROIs) in which B was measured relative to $A$. The three measurements of test object $C$, as referenced to $\mathrm{A}$ and $\mathrm{B}$, agree as well, when the measurements are assumed correct at the mean range $(5.8 \mathrm{~cm})$ of those measurements. For $\mathrm{C}$ relative to $\mathrm{A}, \alpha_{f m}=0.86$ and $\alpha_{f z} z+\alpha_{f 0}=0.85$ and for $\mathrm{C}$ relative to $\mathrm{B}, \alpha_{f m}=0.87$. Ideally, there would be no depth dependence of 


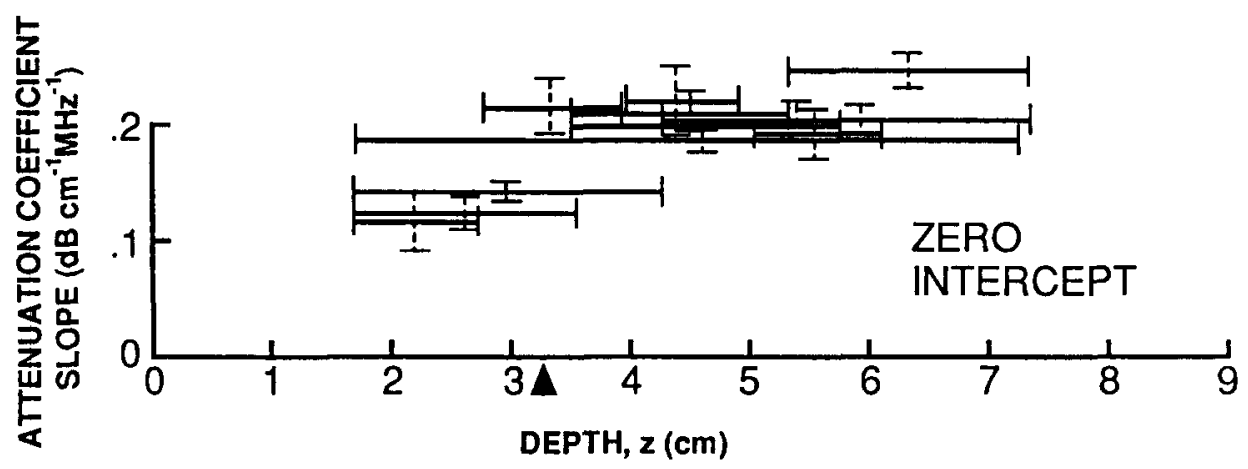

(a)

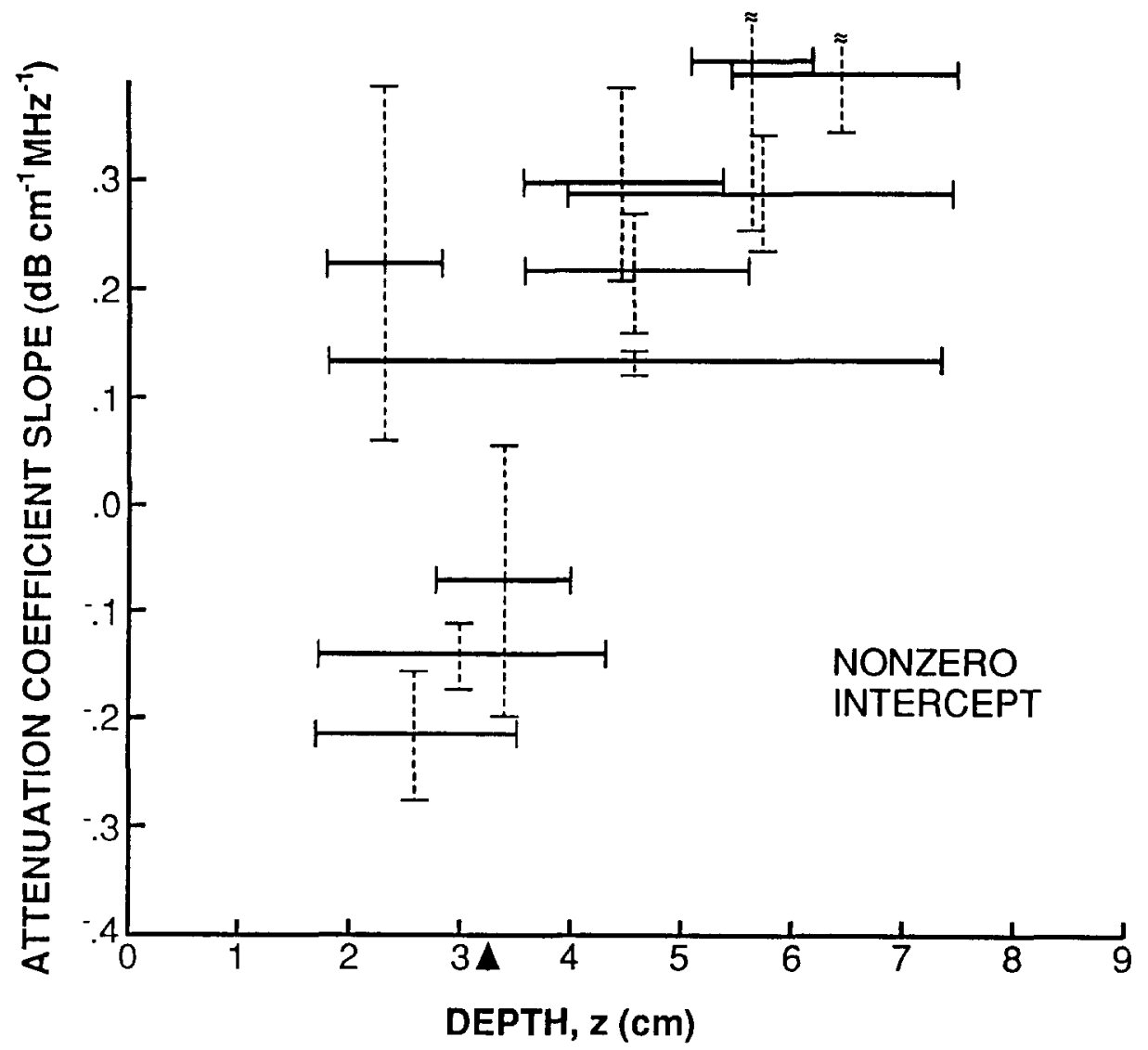

(b)

Fig. 1. Measured attenuation coefficient slope $\alpha_{f}$ is shown as a function of range (distance from the transducer) for a tissue-mimicking test object with an average $\alpha_{f}$ of $0.86 \mathrm{~dB} \mathrm{~cm}^{-1} \mathrm{MHz}^{-1}$. They are measured with: (a) the zero-intercept (single parameter) model; (b) the slope and intercept model. The range over which the measurement was made is shown by the horizontal line; the Standard Error of the Mean (SEM) of the sample of multiple measurements is given by the height of the brackets at the end of each line. Note the SEM is much smaller for

longer sample volumes. The location of the transducer focal point is indicated by the arrow on the abcissa.

the measurement of attenuation coefficient per unit frequency of one homogeneous test object relative to another. The depth dependencies observed, while rather small, indicate some failure from predictable behavior and suggest caution in analysis of the in vivo data. There may have been multiple scattering effects, small imperfections in the modeled dependence of attenuation as a function of frequency or effects of nonlinear propagation in tissue, which we were unable to demonstrate conclusively in a series of attenuation coefficient measurements by backscatter at different output settings. More likely in our commercial real time system were effects of subtle nonlinearities in the system electronics, which were not considered critical in early tests. The selection of the assumed point of zero error in the depth dependent measure- 
Table 1. Test object attenuation coefficient slope $\alpha_{f}$ and its mean value over all depth ranges of measurement, $\alpha_{f m}$ (both in $\mathrm{dB} \mathrm{cm}^{-1} \mathrm{MHz}^{-1}$ ), by each of two backscattering methods, the zero-intercept method and the dual parameter method.

\begin{tabular}{|c|c|c|c|c|c|c|c|c|c|c|}
\hline \multirow{2}{*}{$\begin{array}{c}\text { Test } \\
\text { object }\end{array}$} & \multirow[b]{2}{*}{ Nominal } & \multirow{2}{*}{$\begin{array}{c}\text { Accepted } \\
\alpha_{f}\end{array}$} & \multicolumn{4}{|c|}{$\begin{array}{c}\text { Zero-intercept }{ }^{\dagger} \\
(\operatorname{Re} A \text { at } 0.58)\end{array}$} & \multicolumn{2}{|c|}{$\begin{array}{c}\text { Dualł } \\
\text { parameter }\end{array}$} & \multirow{2}{*}{$\begin{array}{c}\text { Mean range } \\
z_{m}(\mathrm{~cm})\end{array}$} & \multirow{2}{*}{$\begin{array}{c}\text { Transmis'n } \\
\alpha_{f}\end{array}$} \\
\hline & & & $\alpha_{f m}$ & SEM & $\alpha_{f z}$ & $\alpha_{f 0}$ & $\alpha_{f m}$ & SEM & & \\
\hline A & 0.5 & 0.58 & & & & & & & & 0.65 \\
\hline B & 0.3 & 0.26 & 0.27 & 0.012 & 0.023 & 0.16 & 0.323 & 0.081 & 4.5 & 0.22 \\
\hline C & 0.7 & 0.86 & 0.86 & 0.012 & 0.012 & 0.78 & 0.917 & 0.066 & 5.8 & \\
\hline$C \operatorname{Re} B$ & & 0.86 & 0.87 & & & & & & 5.7 & \\
\hline
\end{tabular}

Given also for $\alpha_{f m}$ is the standard error of the mean (SEM). Given for the zero-intercept method only are the weighted least squares fit coefficients of $\alpha_{f}$ as a function of range $z$ in: $\alpha_{f}=\alpha_{f z} z+\alpha_{f 0}$, with the correction slope, $\alpha_{f z}$, in $\mathrm{dB} \mathrm{cm}^{-2} \mathrm{MHz}^{-1}$. $z$ is the mean depth of each measurement region (ROI). The value used in all further calculations is the "accepted" value, taken as the mean of the nominal value and our measured value obtained by the through transmission substitution technique. The zero-intercept $\dagger$ and dual parameter $\ddagger$ backscattered attenuation values were measured relative to test object $\mathrm{A}$ as the reference test object, at $0.58 \mathrm{~dB} \mathrm{~cm}^{-1} \mathrm{MHz}^{-1}$, except in the last row, where B was employed as the reference test object. The mean range, $z_{m}$, of the regions of interest for the backscatter measurements is also given.

ments was appropriate for comparing the mean and the depth dependent measurements, and somewhat arbitrary for comparison of the depth dependent backscatter and accepted values of $\alpha_{f}$ for test object B. Some additional confidence in the measurement technique, particularly in the relative attenuation of test objects $\mathrm{A}$ and $\mathrm{B}$, is provided by the agreement of the backscatter measurements on test object $\mathrm{C}$ relative to both A and B, measured over the same depth ranges. There still remains an uncertainty of approximately $0.08 \mathrm{~dB}$ in the absolute attenuation coefficient slopes, which are all reported relative to either test objects A or B.

Test object B replaced A as the reference for subject studies when the gel under the entrance window of A became cracked from the repeated scanning. To prevent desiccation of the water and other volatiles in the test object, a several millimeter thick layer of fat-equivalent, mineral oil-filled block-copolymer lay just below the urethane scanning window of test object A. This layer was found to be in convex bulges, which undoubtedly varied the beam focusing depending on the location of the transducer. This caused some undefined amount of the reported variation in the data referenced to test object $A$.

The first 178 examinations examined by an experienced technologist with the Mark 100 system are reported. All studies were performed with informed consent of the mothers, under protocols approved by the institutional review board. To evaluate the need for possible depth corrections in the fetal liver data, depth corrections were applied based on those in Table 1 . An assumption was made that the depth dependence was linearly proportional to the relative difference between the measured fetal and the reference test object attenuation coefficients. For exam- ple, when the measured fetal $\alpha_{f}$ was greater than that of the reference test object $\mathrm{A}$, a depth dependence was applied of

$$
\alpha_{f z}=\alpha_{f z}(\mathrm{C} \text { re A })^{*}\left(\alpha_{f}(F)-\alpha_{f}(A)\right) /\left(\alpha_{f}(\mathrm{C})-\alpha_{f}(\mathrm{~B})\right)
$$

This did not consistently improve the coefficient of determination of measured fetal liver attenuation coefficient as a function of gestational age and another approach was investigated.

It has been indicated that theoretical diffraction corrections or reference test object-based diffraction corrections leave significant, unknown depth dependencies in the measured $\alpha_{f}$, unless the reference material is the same scatterer as the tissue measured (Robinson et al. 1984; Cespedes and Ophir 1989). To determine if the corrections described above were adequate, a series of depth correction slopes, $\alpha_{f z}$, was fitted to the in vivo attenuation coefficient data. These slopes were stepped in increments of $0.005 \mathrm{~dB}$ $\mathrm{cm}^{-2} \mathrm{MHz}^{-1}$. It was assumed that the true measured attenuation coefficient slope was:

$$
\alpha_{f}=\alpha_{f \mathrm{calc}}+\alpha_{f z}\left(z-z_{x 0}\right)
$$

$\alpha_{\text {fcalc }}$ is the $\alpha_{f}$ without any further depth correction, $z$ is the midrange of the ROI and $z_{x 0}$ is the range at which $\alpha_{f \text { calc }}$ is known or assumed to be accurate. The weighted linear regression of $\alpha_{f}$ was calculated as a function of gestational age, with the weighting based on the experimental standard deviation of the measurements for each examination (Bevington 1969). For the most recent, 100-case sample, the calculated coefficient of variation, $R^{2}$, was plotted in Fig. 2 as a smooth function of $\alpha_{f z}$. It is clear that $R^{2}$ is a smooth 


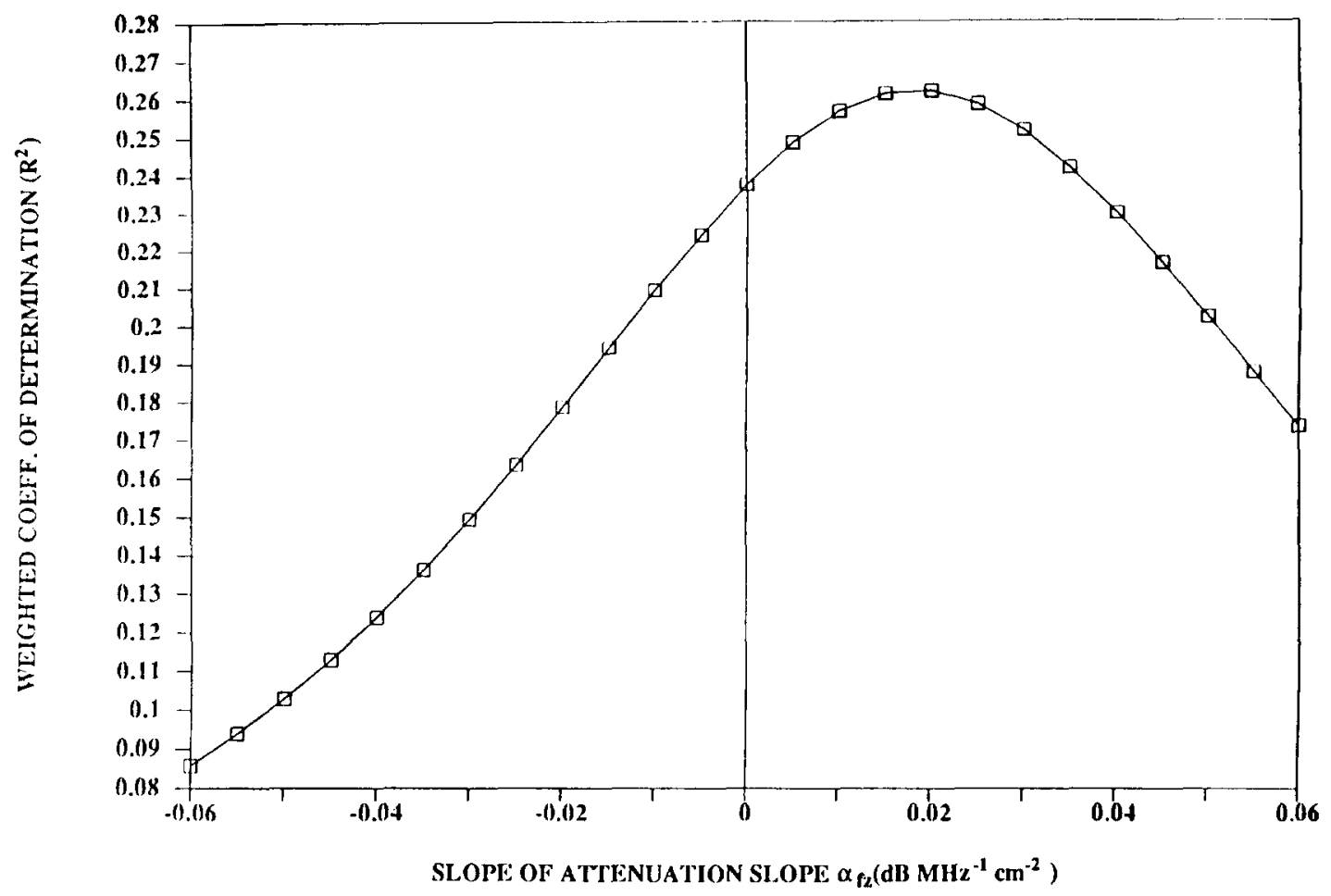

Fig. 2. Empirical determination of the correction to obtain a depth-independent attenuation measure in fetal liver. The coefficient of determination, $R^{2}$, for the weighted linear regression of attenuation slope $\alpha_{f}$ and gestational age, is plotted as a function of the depth-dependent correction $\alpha_{f z}$. A single maximum is exhibited in this analysis for 100 subjects referenced to test object $\mathrm{B}$.

function of $\alpha_{f z}$, and we believe that selection of $\alpha_{f z}$ $=0.020$ at the maximum of $R^{2}$ provided an accurate depth correction for normal fetal liver, with measurements referenced to test object $B$. This maximization of $R^{2}$ essentially did the same thing as performing a weighted multivariate linear regression analysis on the same two variables, depth and gestational age. The same type of $R^{2}$ maximization plot was equally smooth for data from the 78 subjects with test object $\mathrm{A}$, in which $\alpha_{f z}=0.035$ was found to be the appropriate correction. Subject data referenced to test objects $A$ and $B$ were found to have essentially the same average attenuation coefficient when $z_{x 0}=7.0 \mathrm{~cm}$, so that value was assumed for $z_{x 0}$. The mean depths of the ROIs in those two subject groups, A and B, were 6.9 and $7.1 \mathrm{~cm}$, respectively. Thus we believe we have obtained the appropriate depth dependent correction for normal fetal liver with this specific scanhead, and possibly electronics, and have met the criteria for accurate measurements as expressed by Robinson et al. (1984) and Cespedes and Ophir (1989).

While there has been some question about the importance of the abdominal wall and other tissues overlying the organ of interest (Mountford and Wells 1972), it is clear that maternal fat does affect the diffraction correction of a focused ultrasound beam significantly. Carpenter et al. (1988) have shown significant effects of subcutaneous fat thickness with the spheroidal hard-capsule on an end-fire scan head and we probably have similar, slightly reduced effects with the hard, cylindrical window on the ATL scanhead. In a small population, data for possible corrections for overlying tissues were obtained in the form of the thicknesses of various maternal tissue layers lying between the ultrasound transducer and the fetal lung and liver (Carson et al. 1989a, 1989b). This data also had relevance to estimates of ultrasound exposures in vivo for purposes of safety evaluation. The thicknesses of the subcutaneous fat and total abdominal wall were found to correlate well with maternal weight. In unweighted, multivariate linear regressions, inclusion of maternal weight did not significantly improve the correlation of $\alpha_{f}$ with gestational age over that obtained with just the depth correction.

\section{RESULTS}

In subject group $\mathrm{B}$, the attenuation coefficient slope from linear regression was:

$$
\begin{aligned}
\alpha_{f}\left(\mathrm{~dB} \mathrm{~cm}^{-1} \mathrm{MHz}^{-1}\right)= & 0.0086( \pm 0.0003) \\
& \times \mathrm{AGE}(\mathrm{wk})+0.209( \pm 0.071),
\end{aligned}
$$


with $R^{2}=0.26$ and the $F$-test probability $p<0.00001$ that thi slope was zero. See Fig. 3a for data acquired with reference test object $\mathrm{B}$, the $0.26 \mathrm{~dB} \mathrm{~cm}^{-1} \mathrm{MHz}^{-1}$ test object. Shown also are the weighted least squares fit regression line, the $95 \%$ confidence limits for that regression line and the $95 \%$ prediction limits for future data points (the outer lines). Both sets of limits assume only statistical, not systematic, errors. Data and the statistical curves are shown in Fig. $3 b$ for the combination of studies referenced to test objects $A$ and $\mathrm{B}$. In the 78 cases referenced to test object A, the regression fit was:

$$
\begin{aligned}
\alpha_{f}\left(\mathrm{~dB} \mathrm{~cm}^{-1} \mathrm{MHz}^{-1}\right)= & 0.0083( \pm 0.0002) \\
& \times \mathrm{AGE}(\mathrm{wk})+0.222( \pm 0.074),
\end{aligned}
$$

with a coefficient of determination $R^{2}=0.20$ and $p$ $<0.0001$. With the crossover point, $z_{x 0}$, of the depth dependent attenuation chosen at $7 \mathrm{~cm}$, the regression

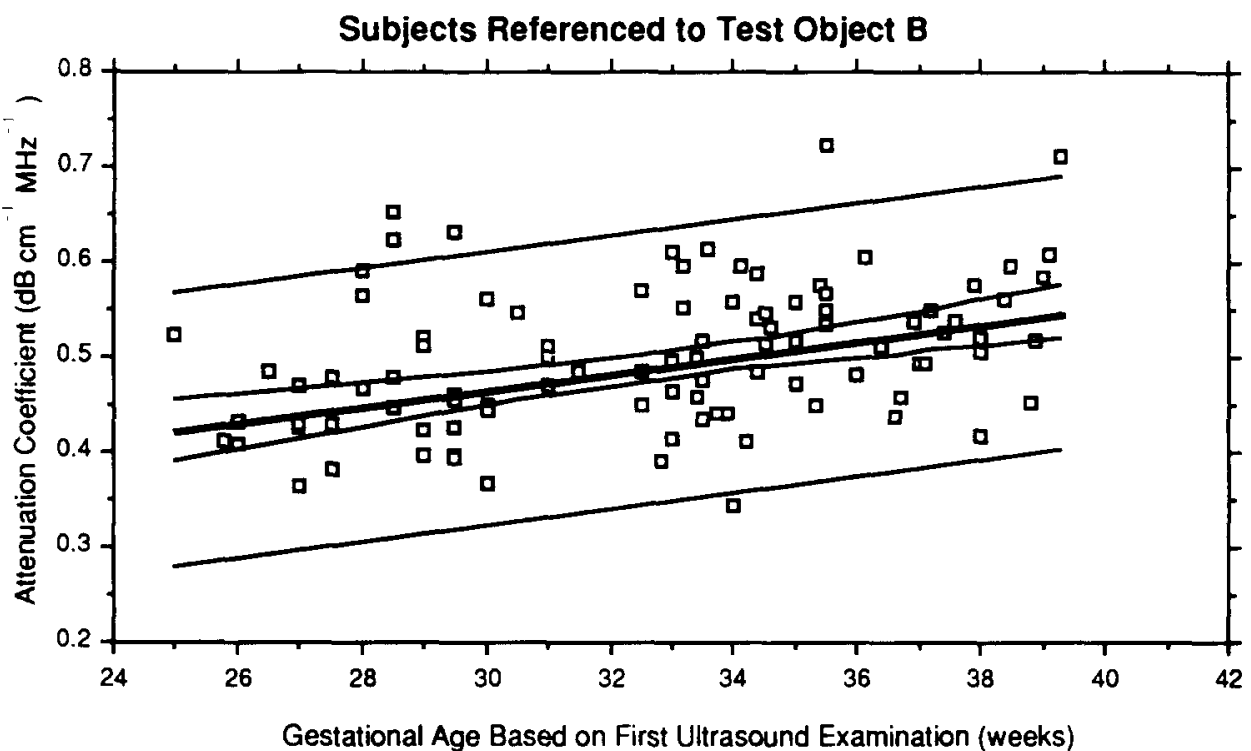

(a)

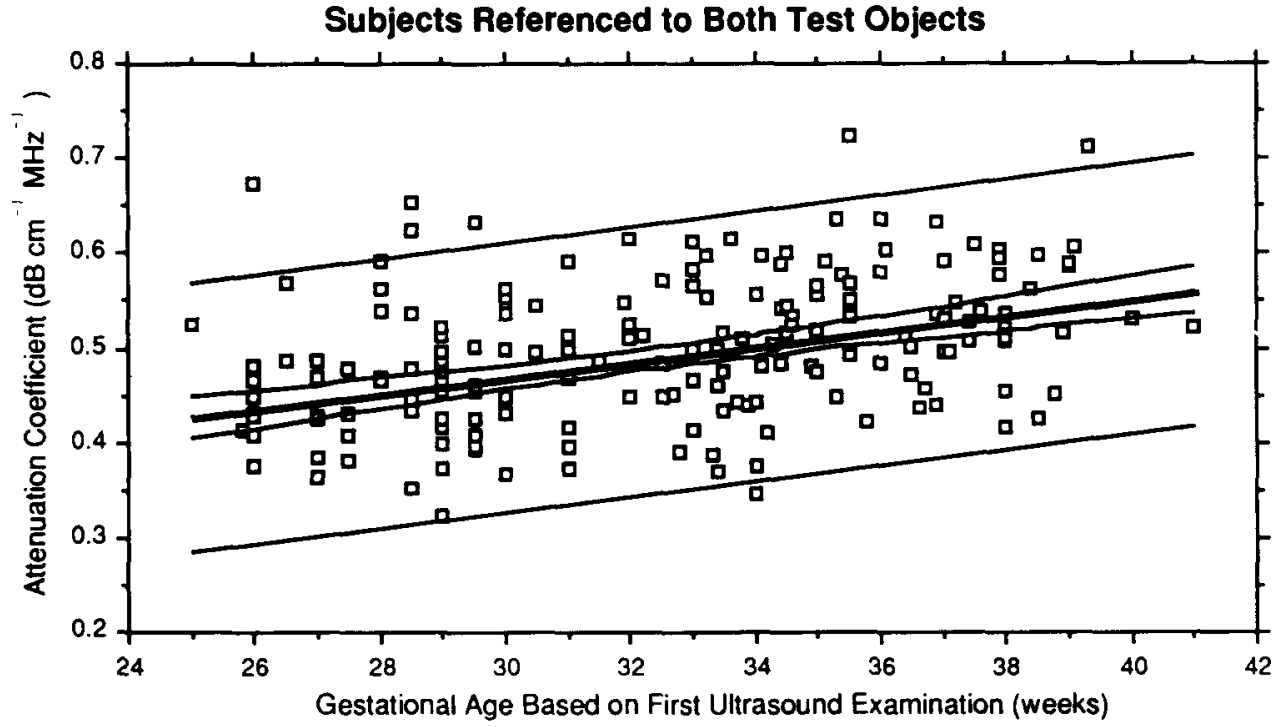

(b)

Fig. 3. Measured attenuation coefficient slope $\alpha_{f}$ for fetal livers in vivo as a function of gestational age. (a) 100 subjects referenced to test object B. (b) 178 subjects referenced to A or B. In each graph, the central bold line shows the weighted least squares fit regression. The first pair of curves on either side show the $95 \%$ confidence limits for that regression line and the outer pair of lines show the prediction limits within which $95 \%$ of future data points should fall. 
lines for data from test objects A and B each gave $\alpha_{f}$ $=0.49 \mathrm{~dB} \mathrm{~cm}^{-1} \mathrm{MHz}^{-1}$, at 33 weeks. In the combination, totaling 178 cases, the regression fit then was:

$$
\begin{aligned}
\alpha_{f}\left(\mathrm{~dB} \mathrm{~cm}^{-1} \mathrm{MHz}^{-1}\right)= & 0.0084( \pm 0.0002) \\
& \times \operatorname{AGE}(\mathrm{wk})+0.227( \pm 0.072),
\end{aligned}
$$

with a coefficient of determination $R^{2}=0.22$.

Gestational age was determined from the gestational age by ultrasound at the first examination plus the difference in dates between any given ultrasound examination and the first one. Similar results were obtained from backward adjustment from the clinical estimates at the time of delivery. Analysis of $\alpha_{f}$ relative to abdominal circumference gave coefficients of determination which were almost as good. This is relevant, since there is some added variation in the data due to necessary reliance on separate measurements for each exam of an individual fetus and since the addition of liver glycogen is proposed as the main reason for rapid growth in abdominal circumference near term. Early analyses of the changes in $\alpha_{f}$ in the serial studies on individual subjects showed similar increases with gestational age, but did not show a dramatic reduction in variance of the increase. Fetal depths did vary over only a surprisingly small range
(Carson et al. 1989b). Multivariate analysis of $\alpha_{f}$, as a function of gestational age and visually-determined lung echogenicity relative to liver, provided a small, but possibly insignificant increase in the coefficient of determination. Further analysis will be required to make any positive statement.

\section{DISCUSSION}

In fetal liver analyses on the same population there continues to be an increase in the attenuation coefficient of $26 \%$ over the full range of gestational ages studied, 26-40 weeks, and over the full range of abdominal circumferences. This statistically significant increase ( $p<0.0001$ that the slope was zero) is consistent with the change in attenuation coefficient expected from the increased glycogen content of the liver during this range of gestational age (Shelly 1961) and from the measured attenuation coefficient effects of glycogen in liver (Parker et al. 1988). Figure 4 (from Shelly) shows a rather gradual, almost linear rise in glycogen content for rhesus monkeys, the curve assumed here for humans. Sheep and dogs appear to rise more steeply to higher concentrations. A later curve measured for humans (Shelly and Neligan 1966) appears to us less reliable, because much of the prenatal data was obtained shortly after premature

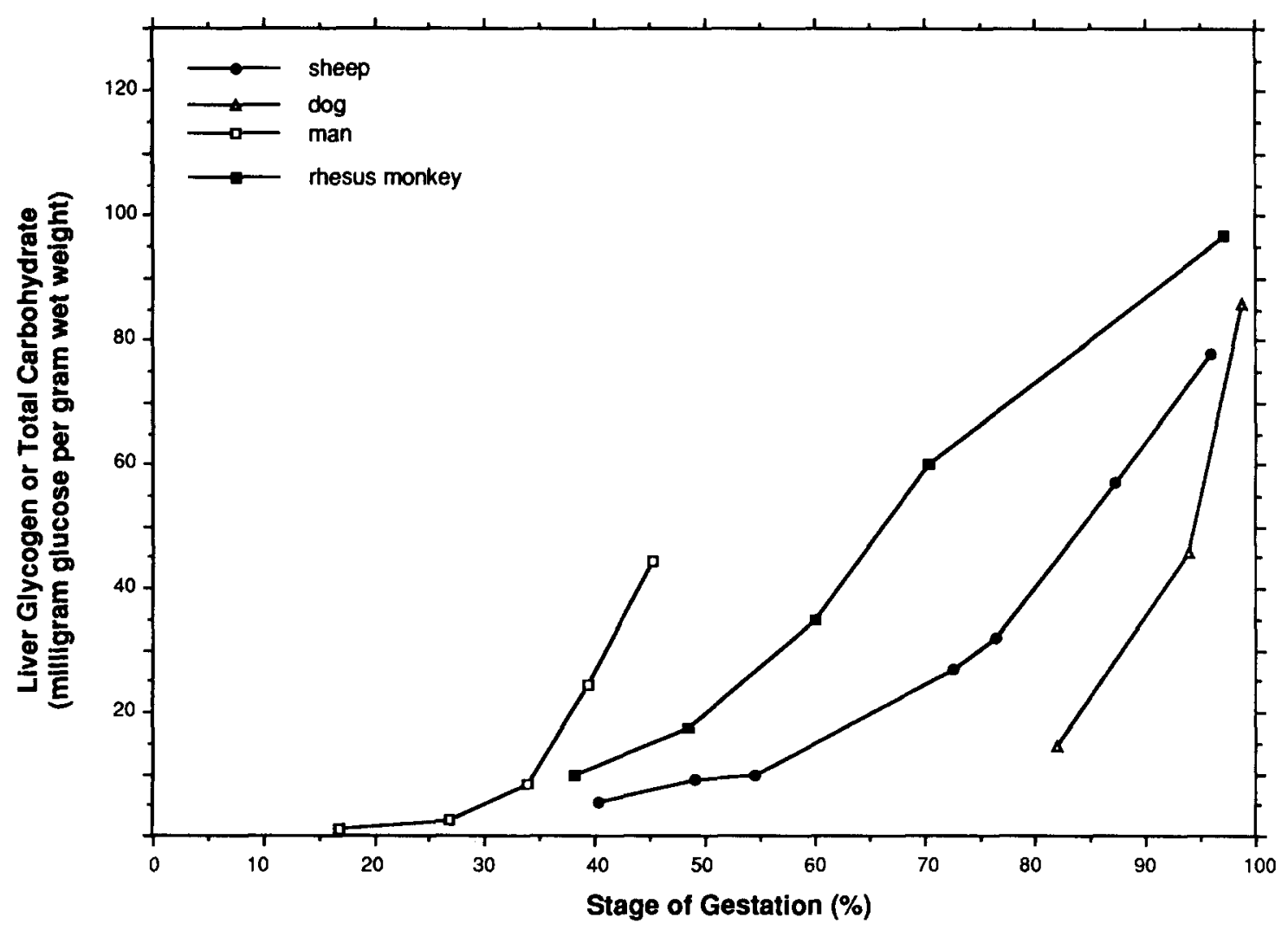

Fig. 4. Fetal liver glycogen in several mammalian species. Redrawn from Shelly (1961). 
birth and death, which would tend to bias the prenatal data toward lower glycogen content. That 1966 human curve does start rising later at 37 weeks. The relative lack of good data on humans and even higher animals is indicative of the invasiveness of current procedures and the need for better measurement methods. Parker et al. measured the added attenuation in liver homogenate to be linear with concentration, such that the modest estimate of $50 \mathrm{mg} / \mathrm{g}$ increase in glycogen from 26-39 weeks would increase the attenuation coefficient at $5 \mathrm{MHz}$ by $20 \%$ or 0.096 $\mathrm{dB} \mathrm{cm} \mathrm{cm}^{-1} \mathrm{MHz}^{-1}$. Parker's results were obtained without adding additional water to the liver homogenate. There is uncertainty as to whether water is added and removed in vivo with glycogen storage and depletion, to maintain a constant proportion of water (the wet hypothesis) or without additional water as a volumeefficient means of energy storage (the dry hypothesis). Their most recent work indicates glycogen is stored with approximately half the usual water content (Tuthill et al. 1989).

\section{CONCLUSIONS}

Analysis of the in vivo attenuation data shows a statistically significant change in ultrasound attenuation coefficient slope by our fetal liver population with increased gestational age. The measured increase in $\alpha_{f}$ by fetal livers is consistent with the hypothesis that such would be observed due to glycogen storage. However, the $26 \%$ change is not large enough to be of clinical use given the current large variance of individual measurements. The attenuation coefficient slope may well prove useful, however, when more precise measures are implemented, including: averaging of more data from independent views; using wider bandwidths; using corrections for phase aberrations by overlying tissue and improved diffraction corrections; automating the elimination of specular echoes and other inhomogeneities in the liver data; and analysis of attenuation in combination with other measures such as scatterer size and echogenicity. Liver volume may be as good as an indicator of rapid glycogen changes, but it is not measured particularly precisely at present and is an indirect measure. If glycogen changes are accompanied by a proportionate change in water content, say 2.3 parts water to one part glycogen, then unusual liver volume changes should be reasonable indicators of glycogen changes, as would occur in the onset of many cases of intrauterine growth retardation (IUGR). If glycogen storage involves very little addition of water, as suggested by the high changes in ultrasound attenuation coefficient slope reported here, then ultrasound attenua- tion may become the best noninvasive measure of fetal glycogen content or changes therein. A large reduction in liver attenuation coefficient recently observed between prenatal and postnatal studies in humans (Carson et al. 1989c) is consistent with the large drop in liver solids from the depletion of glycogen associated with birth (Shelly 1961). That observation further supports the glycogen explanation of changes in liver attenuation coefficient and modest optimism that a noninvasive ultrasound measure of glycogen content can be developed.

Acknowledgements-This work was supported in part from PHS Grant 5R01-HD17243, National Institute for Human Growth and Development. Many of the early human examinations were performed by sonographers, Athena Madonis, RDMS and Kathy Absalom, RDMS. Ms. Margery Rudd performed patient and data management and Fred Bookstein, Ph.D., provided early statistical advice and design. Drs. Peyton Bland, William Bowerman, Dietrich Roloff, William Martel, Terry Silver, William Rayburn, Robert Hayashi, Jonathan Rubin, Caroline Blane and Alan Compton helped establish and conduct the fetal maturity project which included this study.

\section{REFERENCES}

Bevington, P. R. Data reduction and error analysis for the physical sciences. New York: McGraw-Hill Book Co.; 1969:Ch. 6.

Carpenter, D.; Kossoff, G., Robinson, D. S.T.A.R.S.: A technique to reduce beam aberrations due to the subcutaneous tissue layers. J. Ultras. Med. 7:S87 (abstr.); 1988.

Carson, P. L.; Meyer, C. R.; Wang, H. Attenuation estimation with a fixed intercept. Ultrason. Imaging 8:31 (abstr.); 1986.

Carson, P. L.; Meyer, C. R.; Chiang, E. H.; Faix, R. G.; Marks, T. I. Increasing ultrasound attenuation coefficient in the fetal liver as a function of gestational age. J. Ultras. Med. 7:S130 (abstr.); 1988.

Carson, P. L.; Rubin, J. M.; Chiang, E. H. Constant-soft tissue distance model in pregnancies. Ultrasound Med. Biol. 15 (Sup. 1):27-29; 1989a.

Carson, P. L.; Rubin, J. M; Chiang, E. H. Fetal depth and ultrasound path lengths through overlying tissues. Ultrasound Med. Biol. 15:629-639; 1989b.

Carson, P. L.; Chiang, E. H.; Rubin, J. M.; Meyer, C. R.; Andersen, H. F. Pre-to-postnatal reduction in ultrasound attenuation coefficient of the liver. Ultrasound Med. Biol. [1989c].

Cespedes, I.; Ophir, J. Estimation of acoustic attenuation with axial beam translation: Simulation and experiment. Ultrasound Med. Biol. Submitted for publication [1989].

Cloostermans, M. J. T. M.; Thijssen, J. M. A beam corrected estimation of the frequency dependent attenuation of biological tissues from backscattered ultrasound. Ultrason. Imaging 5:136-147; 1983.

Duerinckx, A. J.; Aufrichtig, D.; Rosenberg, K.; Hoefs, J. C.; Ferrari. L. A.; Cole-Beuglet, C. The importance of fat in the estimation of acoustic attenuation in liver with a narrow-band amplitude estimator. J. Ultras. Med. 5:S1 19 (abstr.); 1986.

Duerinckx, A.; Rosenberg, K.; Hoefs, J.; Aufrichtig, D.; ColeBeuglet, C.; Kanel, G.; Lottenberg, S.; Ferrari, L. A. In vivo acoustic attenuation in liver: Correlations with blood tests and histology. Ultrasound Med. Biol. 14:405-413; 1988.

Goss, S. A.; Frizzell, L. A.; Dunn, F. Ultrasonic absorption and attenuation in mammalian tissues. Ultrasound Med. Biol. 5:181-186; 1979 .

Kuc, R.; Schwartz, M. Estimating the acoustic attenuation coeffcient slope for liver from reflected ultrasound signals. IEEE Trans. Sonics and Ultrason. 26:353-362; 1979.

Lin, T.; Ophir, J.; Potter, G. Frequency-dependent ultrasonic dif- 
ferentiation of normal and diffusely diseased liver. J. Acoust. Soc. Am. 82:1131-1138; 1987.

Lizzi, F. L.; King, D. L.; Rorke, M. C.; Hui, J.; Ostromogilsky, M.; Yaremko, M. M.; Feleppa, E. J.; Wai, P. Comparison of theoretical scattering results and ultrasonic data from clinical liver examinations. Ultrasound Med. Biol. 14:377-385; 1988.

Meyer, C. R.; Herron, D. S.; Carson, P. L.; Banjavic, R. A.; Thieme, G. A.; Bookstein, F. L.; Johnson, M. L. Estimation of ultrasonic attenuation and mean backscatterer size via digital signal processing, Ultrason. Imaging 6:13-23; 1984.

Mountford, R. A.; Wells, P. N. T. Ultrasonic liver scanning: The A-scan in normal and cirrhosis. Phys. Med. Biol. 17:261-269; 1972.

Nicholas, D. Evaluation of backscattering coefficients for excised human tissues: Results, interpretation and associated measurements. Ultrasound Med. Biol. 8:17-28; 1982.
Parker, K. J.; Lerner, R. M.; Waag, R. C. Attenuation of ultrasound: Magnitude and frequency dependence for tissue characterization. Radiology 153:785-788; 1984.

Parker, K. J.; Tuthill, T. A.; Baggs, R. B. The role of glycogen and phosphate in ultrasonic attenuation of liver. J. Acoust. Soc. Am. 83:374-378; 1988.

Robinson, D. E.; Wilson, L. S.; Bianchi, T. Beam pattern (diffraction) correction for ultrasonic attenuation measurement. Ultrason. Imaging 6:293-303; 1984

Shelly, H. J. Glycogen reserves and their changes at birth. Brit. Med. Bull. 17:137-143; 1961 .

Shelly, H. J.; Neligan, G. A. Neonatal hypoglycaemia. Br. Med. Bull. 22:34-38; 1966 .

Tuthill, T. A.; Baggs, R. B.; Parker, K. J. Liver glycogen and water storage: Effect on ultrasound attenuation. Ultrason. Imaging 11:138 (abstr.); 1989. 